\title{
Silent thyroiditis following vaccination against COVID-19: report of two cases
}

\author{
M. Capezzone ${ }^{1}$ (I) M. Tosti-Balducci ${ }^{2} \cdot$ E. M. Morabito ${ }^{1} \cdot$ G. P. Caldarelli ${ }^{3} \cdot$ A. Sagnella ${ }^{4} \cdot$ S. Cantara ${ }^{4} \cdot$ M. Alessandri ${ }^{1}$. \\ M. G. Castagna ${ }^{4}$
}

Received: 25 November 2021 / Accepted: 7 December 2021 / Published online: 16 January 2022

(C) Italian Society of Endocrinology (SIE) 2021

\begin{abstract}
Purpose It is well established that thyroiditis and other thyroid disorders can be induced by COVID-19 infection, but there is limited information about the autoimmune/inflammatory syndrome induced by adjuvants (ASIA) after severe acute respiratory syndrome coronavirus 2 (SARS-CoV-2) vaccination. We report two cases of thyrotoxicosis following SARS-CoV-2 vaccine.

Methods and results Two young health care peoples (wife and husband) received a first dose of SARS-CoV-2 vaccine, and few weeks later developed clinical manifestations of thyroid hyperactivity, with increased thyroid hormone levels on thyroid function tests, suppressed thyroid-stimulating hormone and negative antithyroid antibodies, despite being healthy before vaccination. They were diagnosed at the 4th week after first dose of SARS-Cov-2 vaccine as silent thyroiditis and followed without treatment, since their symptoms were not severe. At the 6th week, the patients became wholly asymptomatic and their thyroid function returned to normal.

Conclusions Thyrotoxicosis can occur after SARS-CoV-2 vaccination probably related to silent thyroiditis.
\end{abstract}

Keywords Thyrotoxicosis $\cdot$ COVID vaccine $\cdot$ Adverse vaccine reactions $\cdot$ Silent thyroiditis

\section{Introduction}

Many cases of thyroid dysfunction have been reported in affected patients with mild to moderate severe acute respiratory syndrome related to SARS-CoV-2 infection [1,2]. Angiotensin-converting enzyme 2 (ACE2), the functional receptor for SARS-Cov-2 virus, has been demonstrated to be expressed in thyroid follicular cells, making them a potential target for SARS-COV-2 infection [3]. There are

M. Capezzone

marco.capezzone@uslsudest.toscana.it

1 Present Address: Unit of Endocrinology, Department of Internal Medicine, Misericordia Hospital, 58100 Grosseto, Italy

2 Unit of Nuclear Medicine, Misericordia Hospital, Grosseto, Italy

3 Laboratory Medicine Functional Area, Hospital Misericordia of Grosseto, Grosseto, Italy

4 Section of Endocrinology and Metabolism, Department of Medical, Surgical and Neurological Sciences, University of Siena, Siena, Italy few emerging data about the possible autoimmune/inflammatory syndrome induced by adjuvants (ASIA) following SARS-COV-2 vaccines $[4,5]$.

We reported two cases of silent thyroiditis probably induced by the first dose of mRNA-1273SARS-CoV-2 (Moderna) vaccine.

The aim of this report is to highlight and raise awareness of a potential rare side effect of this vaccine.

\section{Materials and methods}

\section{Patient's findings}

Two patients (wife and husband, 29 and 34 years old, respectively), referred to our Endocrinology Unit for a three-week history of palpitations and weight loss. There was no significant past medical history, including no history of COVID-19 infection, and there was no family history of thyroid disease. Both received the first dose of a SARS-CoV-2 vaccine on September 9, 2021 (COVID-19 Vaccine mRNA-1273 developed by ModernaTx, Inc). Their symptoms started one week after 
administration of the first dose of SARS-CoV-2 vaccine. Their primary care physician ordered the evaluation of thyroid function tests that was performed on October 2, 2021. Thyroid function tests revealed the presence of thyrotoxicosis. Inflammatory markers [erythrocyte sedimentation rate (ESR) and $\mathrm{C}$-reactive protein (CRP)] were normal, and TPOAb and $\mathrm{TgAb}$ were not present.

\section{Results}

\section{Case 1 (husband)}

At the moment of his physical examination, performed on October 2021, 8 , the heart rate was $88 \mathrm{bpm}$. The patient did not report any fever, neck pain or symptoms of upper respiratory infection in the preceding weeks. Thyroid ultrasound revealed a gland of normal volume, with mild hypoechogenicity, diffuse heterogeneous echotexture and decreased color Doppler blood flow signals, without thyroid nodules (Fig. 1a). Thyroid function tests revealed suppressed serum TSH $(<0.003 \mathrm{U} / \mathrm{mL})$ with elevated free thyroxine levels (fT4) (Table 1). Iodine urinary test was negative. Thyroid scintigraphy showed decreased 99mTcpertechnetate uptake.

No specific treatment was initiated, and thyroid function tests were performed several days later.

Thyroid function, performed on October 2021, 12, and on October 2021, 19, returned to normal. The results of the hormonal tests are reported in Table 1.

\section{Case 2 (wife)}

At the moment of physical examination, performed on October 2021,8 , the heart rate was $78 \mathrm{bpm}$. The patient did not report any fever, neck pain or symptoms of upper respiratory infection in the preceding weeks. Thyroid function tests revealed suppressed TSH $(<0.003 \mathrm{U} / \mathrm{mL})$ and normal fT4 and fT3 concentrations (Table 1). Iodine urinary test was negative. Thyroid ultrasound revealed a gland of normal volume with mild hypoechogenicity, diffuse heterogeneous echotexture and decreased color Doppler blood flow signals, with a small thyroid nodule in the left lobe (Fig. 1b). Thyroid scintigraphy showed decreased 99mTcpertechnetate uptake.

No specific treatment was initiated, and thyroid function tests were performed several days later.

Thyroid function, performed on October 2021, 12, and on October 2021, 19, had returned to normal. The results of the hormonal tests are reported in Table 1.

\section{Discussion}

COVID-19 vaccination is the most effective way to protect against COVID-19 illness. Spontaneous reports of suspected adverse reactions are an important source of information for pharmacovigilance activities, as they allow to detect potential safety signals associated with the use of medicines to make them safer, to the benefit of all patients. There are several reports in Literature of people developing thyroid disorders, in particular subacute thyroiditis, after receiving a COVID-19 vaccine [6-8]. In the past, the possibility of developing thyroiditis has been reported as a possible rare complication of Influenza, HPV and HBV vaccination. Genetic predisposition may also play a role, as suggested by the association with HLAB35 and the report of familial subacute thyroiditis cases. In all these cases, association of SAT with the administered vaccines was suggested by patient history (no preceding symptoms of viral infection) and the timing of symptoms onset after the vaccination. In most of these cases, symptoms started after the first dose.

Immune response against the viral antigens following infection or vaccination can cross-react with human tissue antigens that share sequence homology with the virus, resulting in autoimmune reactivity, possibly followed by outright autoimmune disease. Recently it has been reported that SARS-CoV-2 spike protein, nucleoprotein, and membrane proteins all cross-reacted with thyroid peroxidase (TPO) SAT has been described after viral vectors or mRNA vaccinations against COVID-19 suggesting that molecular mimicry might play a significant role in the pathophysiology of these autoimmune responses. SARS-CoV-2 antibodies may promote a mild and transient thyroiditis by cross-reacting with cellular antigens (thyroid peroxidase, TPO) located on the thyroid. Silent (painless) thyroiditis has been recognized over the past decade as a clinical syndrome manifested by transient hyperthyroidism associated with a marked depression of the thyroidal radioactive iodine uptake, often followed by a transient period of hypothyroidism and then by a fully recover to euthyroidism. Silent thyroiditis accounts for approximately to $0.5-5 \%$ of cases of hyperthyroidism and is considered a variant form of chronic autoimmune thyroiditis suggesting that it's a part of the spectrum of thyroid autoimmune diseases. We herein present two cases of silent thyroiditis following SARS-CoV-2 vaccination. After the first dose of the mRNA-1273 COVID-19 vaccine, patients developed thyrotoxicosis which lasted for about four weeks before resolving spontaneously. Patient history, clinical courses of thyroid disfunction and the temporal correlation between the administration of the vaccine and the onset of thyrotoxicosis make it more likely to be a side effect of 

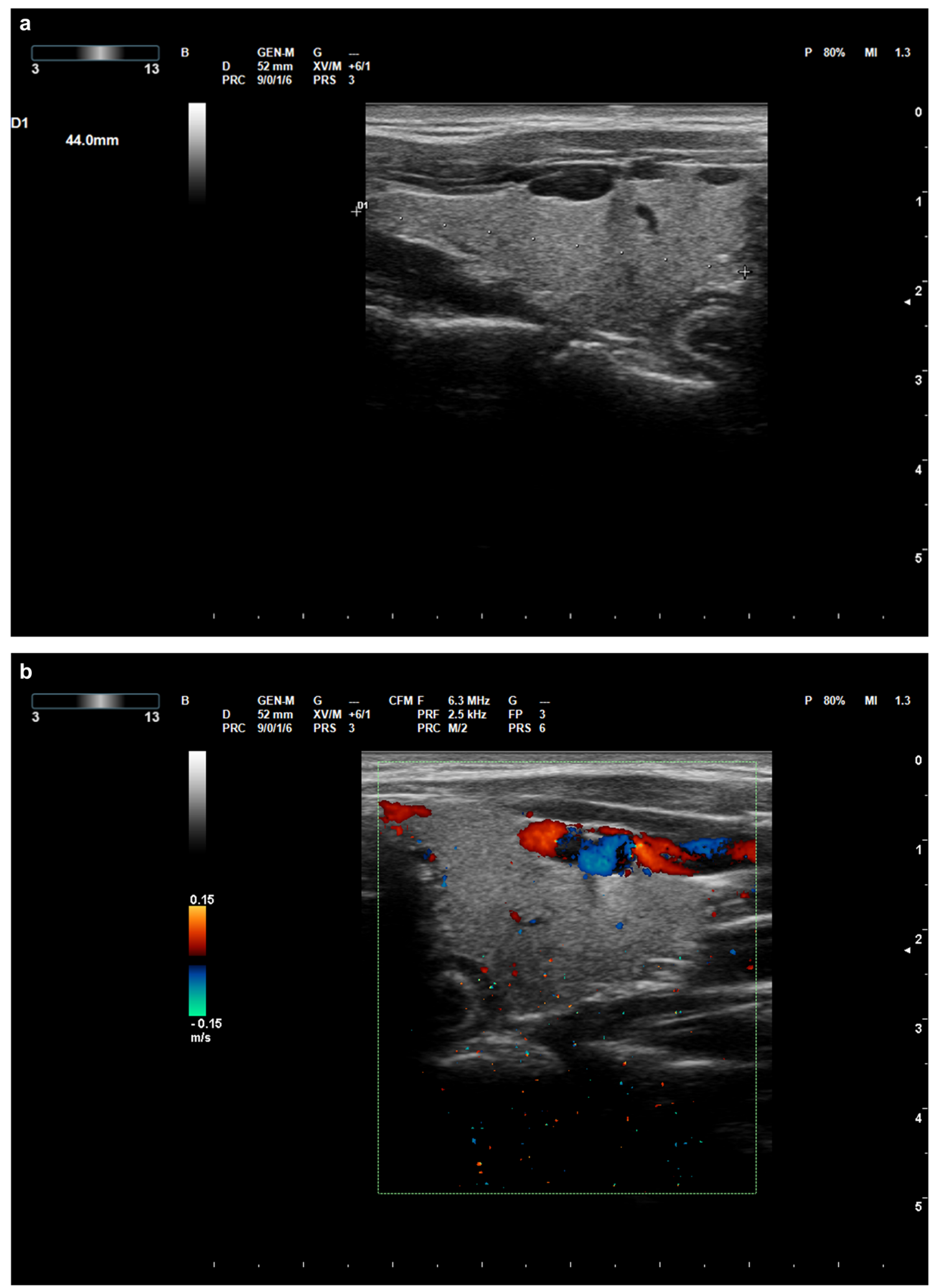

Fig. 1 HYPERLINK "sps:id::fig1|llocator::gr1\|MediaObject::0" a, b Thyroid ultrasound images of case 1 performed during the thyrotoxic phase. $\mathbf{c}, \mathbf{d}$ Thyroid ultrasound images of case 2 performed during the thyrotoxic phase 
Table 1 Laboratory tests results

\begin{tabular}{|c|c|c|c|c|c|c|c|c|c|c|}
\hline & $\begin{array}{l}\text { TSH }(0.2- \\
4.2 \mu \mathrm{UI} / \\
\mathrm{mL})\end{array}$ & $\begin{array}{l}\text { FT3 }(3.1- \\
6.8 \mathrm{pmol} / \mathrm{L})\end{array}$ & $\begin{array}{l}\text { FT4 (12- } \\
22 \mathrm{pmol} / \mathrm{L})\end{array}$ & $\begin{array}{l}\mathrm{TgAb} \\
(<115 \mathrm{IU} / \\
\mathrm{mL})\end{array}$ & $\begin{array}{l}\text { TPOAb } \\
(<34 \mathrm{IU} / \\
\mathrm{mL})\end{array}$ & $\begin{array}{l}\text { TRAb } \\
(<1.7 \text { IU/ } \\
\mathrm{mL})\end{array}$ & $\begin{array}{l}\text { Urinary } \\
\text { iodine } \\
(<300)\end{array}$ & $\begin{array}{l}\text { WBC }(3.5- \\
9.5 \times 10^{3} / \\
\mu \mathrm{L})\end{array}$ & $\begin{array}{l}\text { ESR } \\
(2-25 \mathrm{~mm} / \mathrm{h})\end{array}$ & $\begin{array}{l}\text { CRP } \\
(<0.50 \mathrm{mg} / \\
\mathrm{dL})\end{array}$ \\
\hline \multicolumn{11}{|l|}{ Case 1} \\
\hline 23 days $^{\mathrm{a}}$ & 0.01 & - & 24 & $<0.9$ & $<9$ & $<0.8$ & - & 6.08 & 5 & $<0.06$ \\
\hline 29 days $^{\mathrm{a}}$ & 0.08 & 5.4 & 15.4 & $<0.9$ & $<9$ & $<0.8$ & 98 & - & - & - \\
\hline 33 days $^{\mathrm{a}}$ & 0.3 & 4.9 & 14.8 & $<0.9$ & $<9$ & $<0.8$ & - & - & - & - \\
\hline 40 days $^{\mathrm{a}}$ & 0.6 & 5.7 & 15.7 & $<0.9$ & $<9$ & $<0.8$ & - & - & - & - \\
\hline \multicolumn{11}{|l|}{ Case 2} \\
\hline 23 days $^{\mathrm{a}}$ & 0.03 & 5.8 & 21.7 & $<0.9$ & $<9$ & $<0.8$ & - & 4.43 & 10 & $<0.06$ \\
\hline 29 days $^{\mathrm{a}}$ & 0.2 & 5.4 & 15.4 & $<0.9$ & $<9$ & $<0.8$ & 111 & - & - & - \\
\hline 33 days $^{\mathrm{a}}$ & 1.4 & 4 & 12.2 & $<0.9$ & $<9$ & $<0.8$ & - & - & - & - \\
\hline 40 days $^{\mathrm{a}}$ & 1.6 & 4.7 & 14.4 & $<0.9$ & $<9$ & $<0.8$ & - & - & - & - \\
\hline
\end{tabular}

${ }^{\text {a }}$ Days afterSARS-CoV-2 vaccination

this type of vaccine. The possibility that the two patients accidentally or voluntarily ingested thyroid hormones was excluded on the basis of their anamnesis, except for the intake of substances containing iodine excluded from the negativity of iodine urinary tests.

To our knowledge, only one case of silent thyroiditis has been reported after vaccination against COVID-19. The patient presented with biochemical and imaging features consistent with silent thyroiditis three weeks after vaccination with the ChAdOx1-S (AstraZeneca) vaccine. Similar to our cases, no specific treatment was initiated and, 2 months later, thyroid function tests had returned to normal [7].

The aim is to predict the potential rare side effect possibly related to the administration of this vaccine, although further data are needed on whether the observed association between SARS-CoV-2 vaccination and silent thyroiditis is causal.

When recipients of COVID-19 vaccines complain of symptoms such as fatigue and palpitations, thyrotoxicosis should be considered as a differential diagnosis.

Funding No grants or fellowships have supported the writing of the paper.

\section{Declarations}

Conflict of interest The authors have no conflicts of interest to report. Maria Grazia Castagna is a member of Editorial Board.

Ethical approval All procedures performed were in accordance with the ethical standards of the institutional and/or research committee and with the 1964 Helsinki Declaration and its later amendments or comparable ethical standards. The ethical committee approval is not required for case reports.

Informed consent Informed consent was obtained from all individual partecipants included in the study.

\section{References}

1. Lui DTW, Lee CH, Chow WS, Lee ACH, Tam AR, Fong CHY, Law CY, Leung EKH, To KKW, Tan KCB, Woo YC, Lam CW, Hung IFN, Lam KSL (2021) Thyroid dysfunction in relation to immune profile, disease status, and outcome in 191 patients with COVID-19. J Clin Endocrinol Metab 106:e926-e935. https://doi.org/10.1210/clinem/dgaa813

2. Ippolito S, Dentali F, Tanda ML (2020) SARS-CoV-2: a potential trigger for subacute thyroiditis? Insights from a case report. J Endocrinol Investig 43(8):1171-1172. https://doi.org/10.1007/ s40618-020-01312-7

3. Pal R, Banerjee M (2020) COVID-19 and the endocrine system: exploring the unexplored. J Endocrinol Investig 43:1027-1031. https://doi.org/10.1007/s40618-020-01276-8

4. İremli BG, Şendur SN, Ünlütürk U (2021) Three cases of subacute thyroiditis following SARS-CoV-2 vaccine: postvaccination ASIA syndrome. J Clin Endocrinol Metab 106:2600-2605. https://doi.org/10.1210/clinem/dgab373

5. Vera-Lastra O, Ordinola Navarro A, Cruz Domiguez MP, Medina G, Sánchez Valadez TI, Jara LJ (2021) Two cases of Graves' disease following SARS-CoV-2 vaccination: an autoimmune/inflammatory syndrome induced by adjuvants. Thyroid 31:1436-1439. https://doi.org/10.1089/thy.2021.0142

6. Das L, Bhadada SK, Sood A (2021) Post-COVID-vaccine autoimmune/inflammatory syndrome in response to adjuvants (ASIA syndrome) manifesting as subacute thyroiditis. J Endocrinol Investig 28:1-3. https://doi.org/10.1007/s40618-021-01681-7

7. Siolos A, Gartzonika K, Tigas S (2021) Thyroiditis following vaccination against COVID-19: report of two cases and review of the literature. Metab Open. https://doi.org/10.1016/j.metop. 2021.100136 
8. Pujol A, Gómez LA, Gallegos C, Nicolau J, Sanchís P, González-Freire M, López-González ÁA, Dotres K, Masmiquel L (2021) Thyroid as a target of adjuvant autoimmunity/inflammatory syndrome due to mRNA-based SARS-CoV2 vaccination: from Graves' disease to silent thyroiditis. J Endocrinol Investig 18:1-8. https://doi.org/10. 1007/s40618-021-01707-0

Publisher's Note Springer Nature remains neutral with regard to jurisdictional claims in published maps and institutional affiliations. 